\title{
Cytokeratin alteration in oral leukoplakia and oral squamous cell carcinoma
}

\author{
THOMAS FILLIES ${ }^{1}$, MIRCO JOGSCHIES ${ }^{1}$, JOHANNES KLEINHEINZ ${ }^{1}$, \\ BURKHARD BRANDT ${ }^{2}$, ULRICH JOOS ${ }^{1}$ and HORST BUERGER ${ }^{3}$ \\ ${ }^{1}$ Department of Cranio-Maxillofacial Surgery, University of Münster; ${ }^{2}$ Institute of Tumour Biology, \\ University of Hamburg; ${ }^{3}$ Institute of Pathology, Paderborn, Germany
}

Received February 20, 2007; Accepted May 17, 2007

\begin{abstract}
Intermediate filaments are involved in cell migration and intracellular signal transduction pathways. In a variety of organs, the expression of distinct intermediary filaments are further associated with distinct steps of malignant transformation. In this study, we seeked to define the cytokeratin (Ck) expression pattern in oral leukoplakia and oral squamous cell carcinoma (OSCC). One hundred and ninety-two patients with OSCC, 117 patients with oral leukoplakia without dysplasia (OL) and 23 with oral leukoplakia with dysplasia (squamous intraepithelial neoplasia) (OLD) of the oral cavity were investigated for the immunohistochemical expression of Ck 5-6, Ck 8/18, Ck 1 Ck 10, Ck 14, Ck 19 using the tissue microarray technique. Correlations between clinical features and the expression of cytokeratins were evaluated statistically by $\chi^{2}$ tests. The expression of $\mathrm{Ck} 8 / 18$, Ck 19 and $\mathrm{Ck} 1$ was seen in $3.1 \%(\mathrm{Ck} 8 / 18), 12.5 \%(\mathrm{Ck} 19), 75.4 \%$ (Ck 1) of all leukoplakias, $1.0 \%$ (Ck 8/18), 9.4\% (Ck 19), 76.8\% (Ck 1) in OL, $13.0 \%$ (Ck 8/18), 27.3\% (Ck 19), 68.4\% (Ck 1) in OLD and was significantly associated with the degree of dysplasia (Ck 8/18 p<0.01; Ck 19 p <0.01; Ck 1 p $<0.01)$ and the acquisition of invasive growth properties. The highest frequencies were observed in invasive squamous cell carcinomas. The expression of $\mathrm{Ck} 8 / 18$ and Ck 19 in transformed oral lesions can be regarded as an early feature in the pathogenesis of invasive OSCC. However, the aberrant expression of $\mathrm{Ck}$ 8/18 and $\mathrm{Ck} 19$ in an even higher frequency in invasive carcinomas characterizes the expression of typical glandular cytokeratins as a general progression marker in squamous cell carcinomas. These results can be interpreted as first hints that oral leukoplakias with an expression of $\mathrm{Ck}$ $8 / 18$ or 19 independent of dysplasia, should be resected totally since they might indicate an increased progression potential.
\end{abstract}

Correspondence to: Dr Thomas Fillies, Department of CranioMaxillofacial Surgery, University of Münster, Waldeyerstrasse 30, D-48129 Münster, Germany

E-mail: fillies@uni-muenster.de

Key words: cytokeratin, leukoplakia,oral squamous cell carcinoma, cancer

\section{Introduction}

Cancers of the oropharynx with an incidence of 390,000 per year represent a global health problem. In the last 40 years an increased incidence and mortality of oropharyngeal squamous cell carcinoma are noted world wide (1). An improved understanding of the biology of squamous cell carcinoma (SCC) of the oral cavity and its proposed precursors in some instances such as oral leukoplakia with and without dysplasia could potentially identify prognostic factors allowing tailoring of preventive and surgical strategies.

During the process of malignant transformation of oral epithelium, oral leukoplakia represents one of the first morphologically recognizable epithelial alterations. In this regard, oral leukoplakia is therefore an important lesion for clinical preventive investigation and an improved understanding of molecular changes during the transformation process. Numerous molecular-biological and clinicopathological studies have increased the knowledge on the alteration of cytokeratin expression in tumour biology. Intermediary filaments (IFs) are essential intracellular components, underlying or reflecting distinct cellular properties and differentiation stages in epithelial organs.

Stratified epithelium is usually characterized by the expression of $\mathrm{Ck} 5$ and $\mathrm{Ck}$ 14, mainly in the basal cell layers and is associated with the proliferative potential of these cells $(2,3)$. The intermediary cell layers of keratinizing stratified epithelium shows an additional expression of Ck's 1 and 10, which are regarded as signs of cellular differentiation (2). In contrast, glandular epithelia reveal the expression of cytokeratins Ck's 8/18 and 19 as typical features. The expression of $\mathrm{Ck} 8 / 18$ and the suprabasal expression of $\mathrm{Ck} 19$ seem to be correlated with the premalignant transformation in oral epithelium (4).

The aim of this study was to evaluate alterations in cytokeratin expression in oral leukoplakia without dysplasia, oral leukoplakia with dysplasia (SIN, squamous intraepithelial neoplasia) and oral squamous cell carcinomas (OSCCs) by the use of tissue microarrays and immunohistochemistry.

\section{Materials and methods}

Patients and tumour material. Tissue samples of patients with histologically proven oral leukoplakia $(n=140)$ and squamous 
cell carcinoma ( $n=192)$ treated surgically were eligible for the study. From all available cases new sections were prepared for histological re-evaluation and grading according to the squamous intraepithelial neoplasia concept.

All patients with oral leukoplakia underwent biopsy. Leukoplakia with any grade of dysplasia were treated surgical with resection of the whole lesion with a dysplastic-free margin.

All patients with squamous cell carcinoma underwent surgical treatment including radical resection of the whole tumour with a free histopathological margin of at least $4 \mathrm{~mm}$ from the tumour borders. Selective neck dissection of level I, II, III and V was performed in case of suspect results in preoperative tumour staging by computer tomography or sonography examination or in case of tumour size over $2 \mathrm{~cm}$, bilateral selective neck dissection was performed when the tumour spread the midline [according to the recommendation of Robins et al (5)]. All tumours were classified post-surgically according to the TNM system (6).

Method. Oral leukoplakia specimens of 140 patients and tumour specimens of 192 patients were investigated for the expression of Ck 5/6, Ck 8/18, Ck 1, Ck 10, Ck 14, Ck 19 by means of the tissue microarray (TMA) technique.

For tissue microarray (TMA) construction, two punch biopsies with a diameter of $0.6 \mathrm{~mm}$ from each donor block were taken and transferred into the new acceptor block. TMA construction was performed by using a special tissue microarray instrument (Beecher Instruments, NJ, USA), according to standard protocols $(7,8)$.

Immunohistochemistry was performed on $4-\mu$ m-thick TMA sections. After deparaffinization and rehydration, endogenous peroxidase activity was blocked for $30 \mathrm{~min}$ in methanol containing $0.3 \%$ hydrogen peroxide. After antigen retrieval, a cooling-off period of 20 min preceded the incubation of the primary antibody. Thereafter, the catalyzed signal amplification system (Dako, Glostrup, Denmark) was used for Ck 56, $\mathrm{Ck} 8 / 18, \mathrm{Ck} 1, \mathrm{Ck} 10, \mathrm{Ck} 14, \mathrm{Ck} 19$ staining according to the manufacturer's instructions.

All antibodies were detected by a standard avidin-biotin complex method with a biotinylated rabbit anti-mouse antibody (Dako) and an avidin-biotin complex (Dako). All stainings were developed with diaminobenzidine. Before the slides were mounted, all sections were counterstained for $45 \mathrm{sec}$ with hematoxylin and dehydrated in alcohol and xylene. Appropriate negative controls (obtained by omission of the primary antibody) and positive controls were used throughout.

The expression of Ck 5-6, Ck 8/18, Ck 1, Ck 10, Ck 14, Ck 19 were determined independently by two observers. Both pathologists determined the percentage of positive cells in each core.

The percentage was classified for Ck 5-6, Ck 8/18, Ck 1, Ck 10, Ck 14, Ck 19 in two groups ( 0 , no expression; $1, \geq 1 \%$ positive expression). The mean percentage value of two cores representing one tumour and were used for further evaluation.

Statistical analysis. Statistical analyses were performed by using SPSS (version 12.0; SPSS Inc., USA). Correlations
Table I. Tumour patient cases in this study.

Oral squamous cell carcinoma (OSCC)

\begin{tabular}{ll}
\hline Age (mean) & 59 years (range 31-90 years) \\
Sex & 154 men, 38 women \\
T stadium & $95 \mathrm{~T} 1 ; 82 \mathrm{~T} 2 ; 15 \mathrm{~T} 3-\mathrm{T} 4$ tumours \\
N stadium & 135 lymph node negative and 57 lymph \\
& node positive carcinoma \\
Grading & 44 well-differentiated tumours (G1) \\
& 75 moderately differentiated tumours (G2) \\
& 73 poorly differentiated tumours (G3) \\
\hline
\end{tabular}

Table II. Oral leukoplakia patient cases in this study.

\begin{tabular}{ll}
\hline & \multicolumn{1}{c}{ Oral leukoplakia (OL) } \\
\hline Age (mean) & 54 years (range 22-86 years) \\
Sex & 84 men, 56 women \\
Dysplasia & 117 oral leukoplakia without dysplasia \\
& $9 \mathrm{SIN} \mathrm{I}^{\circ}$ \\
& $7 \mathrm{SIN} \mathrm{II}^{\circ}$ \\
& $7 \mathrm{SIN} \mathrm{III}^{\circ}$
\end{tabular}

SIN, squamous intraepithelial neoplasia.

between clinical features and the expression of cytokeratins were evaluated statistically by $\chi^{2}$ tests.

\section{Results}

The series comprised 192 patients with OSCC, 117 patients with oral leukoplakia and 23 with dysplastic oral leukoplakia. All tumours were classified post-surgically according to the TNM system (6). Clinical and tumour details of the patients with oral squamous cell cancer and leukoplakia are shown in Tables I and II. On average $86 \%$ of all cores could be evaluated for the expression of intermediate filaments.

Immunohistochemical examination showed that $\mathrm{Ck} 8 / 18$, Ck 5, Ck 1, Ck 10, Ck 14, Ck 19 reactivity was confined homogeneously to the cytoplasma of the positive cells. The expression levels did not differ significantly for the vast majority of the cases between the two core biopsies representing one case, indicating a rather homogeneous expression (Fig. 1).

Detectable levels of $\mathrm{Ck} 8 / 18(\mathrm{Ck} 8 / 18 \geq 1 \%$ ) were found in $66.7 \%(120 / 180)$ of the oral SCCs, in $1.0 \%(1 / 105)$ of the oral leukoplakia and $13.0 \%(3 / 23)$ of the oral leukoplakia with dysplasia. Detectable levels of Ck 19 (Ck 19 $\geq 1 \%$ ) were found in $40.6 \%(61 / 150)$ of the investigated tumour specimens, in $9.4 \%(10 / 106)$ of the oral leukoplakia and $27.2 \%(6 / 22)$ of the oral leukoplakia with dysplasia.

Cases of positive cytokeratin $8 / 18$ expression increased from $1.0 \%(1 / 105)$ in leukoplakia to $13.0 \%(3 / 23)$ in leuko- 


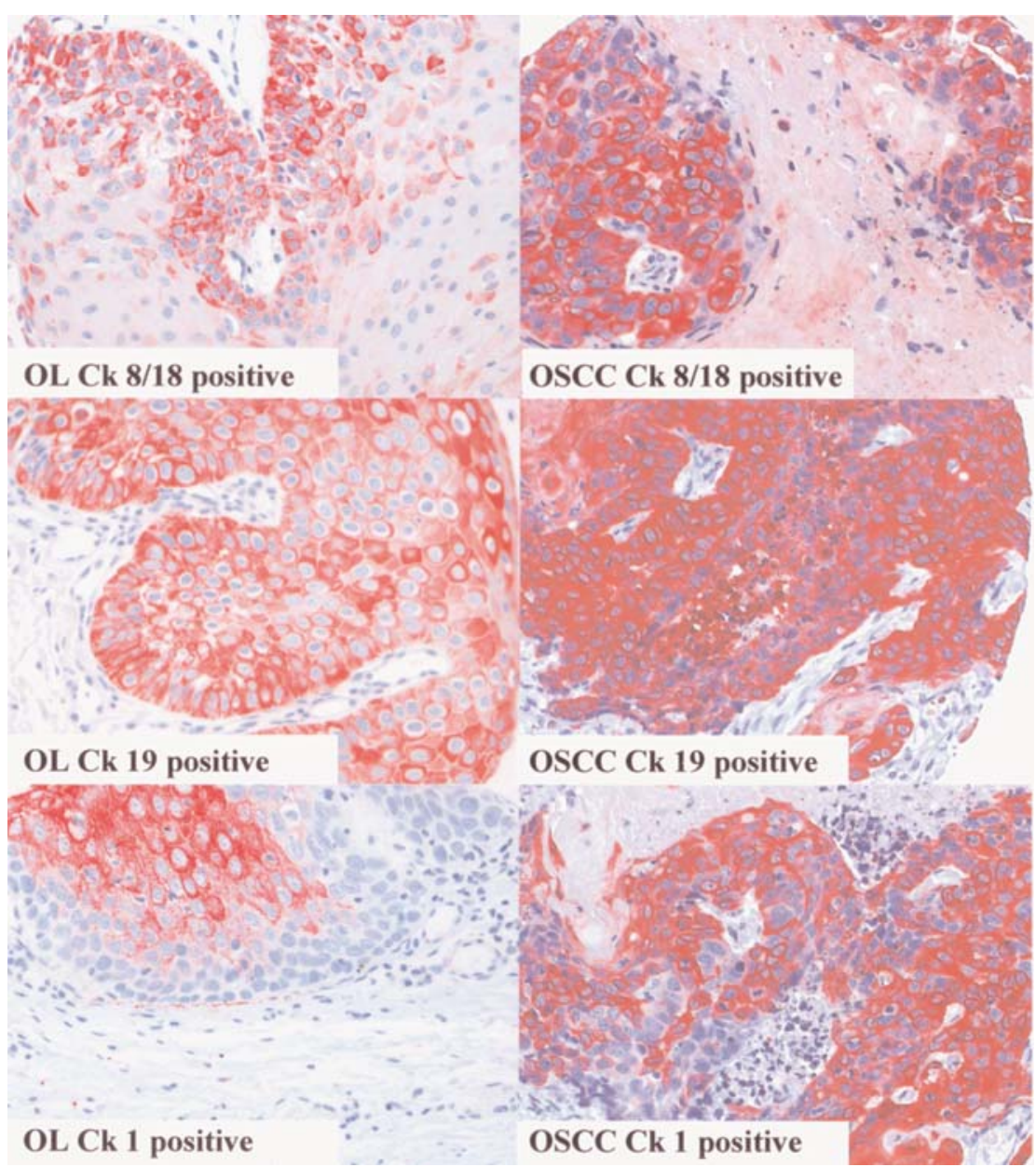

Figure 1. Immunohistochemical staining of OL and OSCC with Ck 8/18, Ck 19 and Ck 1 (left, oral leukoplakia; right, oral squamous cell carcinoma), magnification $\mathrm{x} 20$.

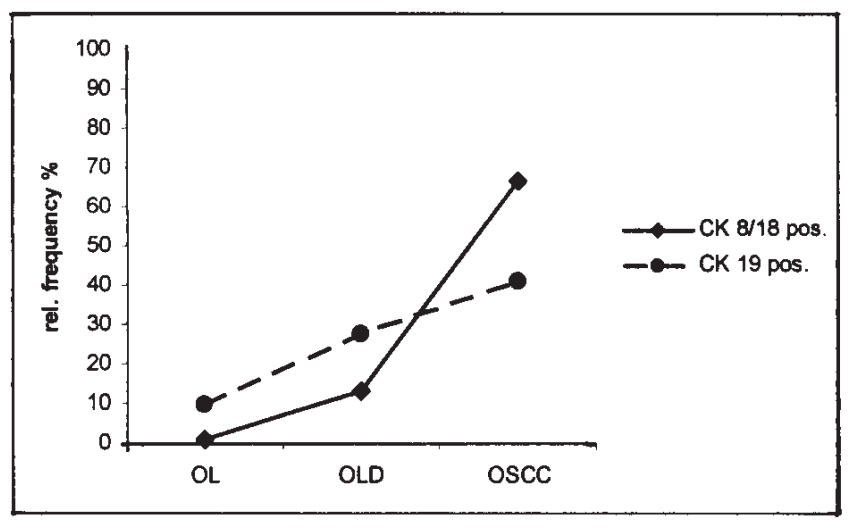

Figure 2. Relative frequency of $\mathrm{Ck} 8 / 18$ and $\mathrm{Ck} 19$ positive cases dependent on the transformation (OL, oral leukoplakia without dysplasia; OLD, oral leukoplakia with dysplasia; OSCC, oral squamous cell carcinoma).

plakia with dysplasia to $66.7 \%(120 / 180)$ in oral squamous cell carcinoma (Fig. 2). The expression of $\mathrm{Ck} 8 / 18$ were significantly correlated in the $\chi^{2}$ test with dysplastic transformation in leukoplakia $(\mathrm{p}<0.01)$ and were significantly higher expressed in OSCCs $(0<0.01)$ (Table III).

The expression of $\mathrm{Ck} 19$ showed likewise an increased number of positive cases dependent on the status of trans- formation (leukoplakia without dysplasia 9.4\% (10/106), leukoplakia with dysplasia $27.2 \%(6 / 22)$ and OSCCs $40.6 \%$ (61/150) (Fig. 2). The expression of Ck 19 were significantly correlated in the $\chi^{2}$ test with dysplastic transformation in leukoplakia ( $\mathrm{p}=0.02)$ and in OSCCs $(\mathrm{p}<0.01)$ (Table III).

Ck 1 expression was significantly reduced in oral squamous cell carcinomas compared to oral leukoplakia $(\mathrm{p}<0.01)$. The expression of Ck 1 was seen in SIN without the degree of dysplasia.

The expression of $\mathrm{Ck}$ 8/18 and $\mathrm{Ck} 19$ revealed no significant correlation. No correlation was found between malignant transformation (leukoplakias vs. invasive carcinomas) and the expression Ck 5/6, Ck 14 and Ck 10 in $\chi^{2}$ test.

\section{Discussion}

The pathogenesis of squamous cell carcinomas is poorly understood. A variety of cell biological markers mainly involved in cell proliferation and apoptosis have been described. In various other entities it could also be shown that the abberant expression of cytokeratins as a main family of intermediate filaments might add additional prognostic significance.

In detail, various studies have shown an association between changes in the intermediate filament expression and altered cellular behaviour $(9,10)$. The aim of this study was 
Table III. Expression profile of cytokeratins.

\begin{tabular}{|c|c|c|c|c|c|c|c|c|c|}
\hline \multirow{2}{*}{$\begin{array}{l}\text { Cytokeratin } \\
\text { antibody }\end{array}$} & \multicolumn{2}{|c|}{$\begin{array}{c}\text { OL } \\
(\mathrm{n}=117)\end{array}$} & \multicolumn{2}{|c|}{$\begin{array}{c}\text { OLD } \\
(\mathrm{n}=23)\end{array}$} & \multicolumn{2}{|c|}{$\begin{array}{c}\text { OSCC } \\
(\mathrm{n}=192)\end{array}$} & \multirow{2}{*}{$\begin{array}{c}\chi^{2} \text {-test } \\
\text { OL vs. OLD }\end{array}$} & \multirow{2}{*}{$\begin{array}{c}\chi^{2} \text {-test } \\
\text { OLD vs. OSCC }\end{array}$} & \multirow{2}{*}{$\begin{array}{c}\chi^{2} \text {-test } \\
\text { OL vs. OLD } \\
\text { vs. OSCC }\end{array}$} \\
\hline & - & + & - & + & - & + & & & \\
\hline Ck 1 & 22 & 73 & 6 & 13 & 83 & 70 & ns & ns & $\mathrm{p}<0.01$ \\
\hline Ck 8/18 & 104 & 1 & 20 & 3 & 60 & 120 & $\mathrm{p}=0.03$ & $\mathrm{p}<0.01$ & $\mathrm{p}<0.01$ \\
\hline Ck 19 & 96 & 10 & 16 & 6 & 89 & 61 & $\mathrm{p}=0.02$ & $\mathrm{~ns}$ & $\mathrm{p}<0.01$ \\
\hline $\mathrm{Ck} 5 / 6$ & 0 & 105 & 0 & 23 & 3 & 178 & $\mathrm{~ns}$ & ns & ns \\
\hline Ck 10 & 66 & 37 & 14 & 8 & 96 & 58 & ns & ns & ns \\
\hline Ck 14 & 0 & 104 & 0 & 23 & 4 & 151 & ns & ns & ns \\
\hline
\end{tabular}

OL, oral leukoplakia without dysplasia; OLD, oral leukoplakia with dysplasia; OSCC, oral squamous cell carcinoma; -, negative; +, positive

to expand on these data, and re-evaluate the progostic relevance in a greater specimen series.

In this study we were able to demonstrate that oral SSCs express Ck 1 in a significantly lower frequency compared to oral leukoplakias $(\mathrm{p}<0.01)$. However, no significant alteration of $\mathrm{Ck} 1$ expression was found within the group of oral leukoplakia dependent on the degrees of histological dysplasia. In contrast, $\mathrm{Ck} 10$ revealed a stable expression in comparison to OL and OSCC. These results are in line with previous studies showing a reduction of $\mathrm{Ck} 1$ in relation to the degree of intraepithelial dysplasia (11). Our results therefore also underline the interpretation that the aberrant expression of $\mathrm{Ck} 1$ is a reflection of epithelial differentiation in the context of a preneoplastic transformation $(12,13)$. In contrast, the presence of $\mathrm{Ck} 10$ might delay tumour development (14). Taken together the loss of $\mathrm{Ck} 1 / 10$ has a potential prognostic value in the appraisal of premalignant oral lesions (15).

Mature, healthy non-keratinizing stratified squamous epithelium of the upper aerodigestive tract normally expresses $\mathrm{Ck} 19$ in the basal layer, but is not characterized by the expression of $\mathrm{Ck} 8 / 18$ (16). Interestingly we were able to demonstrate a high frequency of Ck 8/18 expression in oral leukoplakia. Oral leukoplakia with dysplasia displayed a significantly higher frequency compared to oral leukoplakia without dysplasia, but still a significant difference could be shown compared to oral SCCs (OL vs. OSCC's p $<0.01$; OL vs. OLD $\mathrm{p}=0.03$ ). The interpretation of these finding offers several explanations. The de novo-expression of $\mathrm{Ck} 8 / 18$ in previously negative epithelium during malignant transformation might be regarded as return towards embryonal expression patterns (17). The expression of $\mathrm{Ck} 8$ and 18 could be observed in fetal buccal mucosa and tongue epithelium until 27 weeks of gestation (18). On the other hand the expression of $\mathrm{Ck} 8 / 18$ in association with $\mathrm{Ck} \mathrm{19}$, a previously described stem cell marker, might reflect in accordance with the above mentioned embryonal hypothesis, the multi-potential differentiation patterns of the original tumour stem cells. In a previous study we showed a decreased overall prognosis in relation to the expression of Ck 8/18 (19). Recent studies could show that this cytokeratin pair seems to play an important role in the pathogenesis and progression of OSCCs. The transfection of buccal mucosa cells with a vector for $\mathrm{Ck} 8 / 18$ resulted in a significantly altered cellular morphology and increased cell motility features associated with or being prerequisites for invasive tumour behaviour (20). This was in line with previous experiments in melanoma cells (21). A spontaneous induction of Ck's 8 and 18 expression could be shown in SV40Timmortalized buccal mucosa cells, in tobacco induced leukoplakia and after introduction of v-H-Ras in epidermal mouse keratinocytes (22). The involvement of $\mathrm{Ck} 8 / 18$ in SCC progression could further be demonstrated in chemically induced SCCs in the mouse skin. Whereas SCCs in mouse epidermis were $\mathrm{Ck} 8 / 18$ positive, non-invasive papillomas did not show this feature. Additionally, Ck 8/18 seems to modulate the transformation process and leads to resistance of the Fas induced apoptosis $(23,24)$. However, the underlying disturbed intra- and extracellular regulation mechanisms have not been characterized in detail yet. However, our results show that the altered cytokeratin expression patterns are a rather early event in the pathogenesis of oral SCC's. In line with these findings, further studies have described a low expression of $\mathrm{Ck} 8$ at the healthy side in tumour border regions whereas invasive squamous tumour cells showed a high expression level. Therefore, $\mathrm{Ck} 8 / 18$ is a marker in appraisal of alterated cells in a premaligant stages and early cancer (25-27).

In our investigation, the $\mathrm{Ck} 19$ expression shows similar results to the expression of $\mathrm{Ck} 8 / 18$. We found a significant higher amount of $\mathrm{Ck} 19$ expression dependent on the degree of epithelial transformation (OL vs. OSCC's $\mathrm{p}<0.01$; OL vs. OLD $\mathrm{p}=0.021$ ). Similar results are reported in the literature. The expression of Ck 19 seems to be a common event in dysplastic lesions $(27,28)$ and is mainly interpreted as a marker of dysfunctional epithelial differentiation (29). It is therefore worth speculating that $\mathrm{Ck} 8 / 18$ and $\mathrm{Ck} 19$ are possible markers in the evaluation of the margin after tumour resection in otherwise morphologically normal-appearing epithelium as shown in the past. Investigations of field cancerisation revealed $\mathrm{Ck} 19$ positivity adjacent to $1 \mathrm{~cm}$ of a tumour-free resection margin. Noteworthy the same study showed that $\mathrm{Ck} 8$ expression within this range could be 
observed in up to $30 \%$ of the cases (30). This will additionally support the value of cytokeratin alteration during pathogenesis of oral cancer.

In conclusion, our results in a series of 192 squamous cell carcinomas, 117 leukoplakia and 23 leukoplakia with dysplasia of the oral cavity showed an important change in the cytokeratin expression pattern for $\mathrm{Ck} \mathrm{8/18}$ and 19 in the initiation and progression of squamous cell carcinomas and its precursor lesions. From a clinical point of view it seems advisable to resect oral leukoplakia with an expression of $\mathrm{Ck} 8 / 18$ or 19 independent of dysplasia. This is of special importance against the background that $\mathrm{Ck} 8 / 18$ and $\mathrm{Ck} 19$ positive carcinomas revealed a significantly decreased prognosis.

\section{References}

1. Stewart BW and Kleihues P: Head and Neck Cancer. WHO World Cancer Report, IARC Press, Lyon, 2003.

2. Presland RB and Jurevic RJ: Making sense of the epithelial barrier: what molecular biology and genetics tell us about the functions of oral mucosal and epidermal tissues. J Dent Educ 66: 564-574, 2002.

3. Van der Velden LA, Manni JJ, Ramaekers FC and Kuijpers W: Expression of intermediate filament proteins in benign lesions of the oral mucosa. Eur Arch Otorhinolaryngol 256: 514-519, 1999.

4. Lindberg K and Rheinwald JG: Suprabasal 40 kd keratin (K19) expression as an immunohistologic marker of premalignancy in oral epithelium. Am J Pathol 134: 89-98, 1989.

5. Robbins KT, Clayman G, Levine PA, Medina J, Sessions R, Shaha A, Som P and Wolf GT: American Head and Neck Society; American Academy of Otolaryngology - Head and Neck Surgery: Neck dissection classification update: revisions proposed by the American Head and Neck Society and the American Academy of Otolaryngology-Head and Neck Surgery. Arch Otolaryngol Head Neck Surg 128: 751-758, 2002.

6. Wittekind C, Greene FL, Hutter RVP, Klimpfinger M and Sobin LH (eds): TNM Atlas. 5th edition. Springer Verlag Heidelberg, 2005.

7. Kononen J, Bubendorf L, Kallioniemi A, Barlund M, Schraml P, Leighton S, Torhorst J, Mihatsch MJ, Sauter G and Kallioniemi OP: Tissue microarrays for high-throughput molecular profiling of tumour specimens. Nat Med 4: 844-847, 1998.

8. Packeisen J, Buerger H, Krech R and Boecker W: Tissue microarrays: a new approach for quality control in immunohistochemistry. J Clin Pathol 55: 613-615, 2002.

9. Watanabe S, Ichikawa E, Takahashi H and Otsuka F: Changes of cytokeratin and involucrin expression in squamous cell carcinomas of the skin during progression to malignancy. Br J Dermatol 132: 730-739, 1995.

10. Morgan PR and Su L: Intermediate filaments in oral neoplasia. I. Oral cancer and epithelial dysplasia. Eur J Cancer B Oral Oncol 30B: 160-166, 1994.

11. Bloor BK, Seddon SV and Morgan PR: Gene expression of differentiation-specific keratins in oral epithelial dysplasia and squamous cell carcinoma. Oral Oncol 37: 251-261, 2001.

12. Sawant SS, Naresh KN, D'Cruz A, Ogale SB and Vaidya MM: Demonstration of cytokeratin-5 non-expression in tobacco related oral carcinogenesis-use of reverse transcriptase polymerase chain reaction as a sensitive assay. Oral Oncol 39: 789-795, 2003
13. Cintorino M, Petracca R, Vindigni C, Tripodi SA and Leoncini P: Topography-related expression of individual cytokeratins in normal and pathological (non-neoplastic and neoplastic) human oral mucosa. Virchows Arch A Pathol Anat Histopathol 417: 419-426, 1990.

14. Santos M, Ballestin C, Garcia-Martin R and Jorcano JL: Delays in malignant tumour development in transgenic mice by forced epidermal keratin 10 expression in mouse skin carcinomas. Mol Carcinog 20: 3-9, 1997.

15. Kannan S, Balaram P, Jagadeesh Chandran G, Radhakrishna Pillai M, Mathew B, Nalinakumari KR and Krishnan Nair M: Alterations in expression of terminal differentiation markers of keratinocytes during oral carcinogenesis. Pathobiology 62: 127-133, 1994.

16. Chu PG and Weiss LM: Keratin expression in human tissues and neoplasms. Histopathology 40: 403-439, 2002.

17. Balm AJ, Hageman PC, van Doornewaard MH, Groeneveld EM and Ivanyi D: Cytokeratin 18 expression in squamous cell carcinoma of the head and neck. Eur Arch Otorhinolaryngol 253: 227-233, 1996

18. Vaidya MM, Sawant SS, Borges AM, Naresh NK, Purandare MC and Bhisey AN: Cytokeratin expression in human fetal tongue and buccal mucosa. J Biosci 25: 235-242, 2000.

19. Fillies T, Werkmeister R, Packeisen J, Brandt B, Morin P, Weingart D, Joos U and Buerger H: Cytokeratin 8/18 expression indicates a poor prognosis in squamous cell carcinomas of the oral cavity. BMC Cancer 6: 10, 2006.

20. Raul U, Sawant S, Dange P, Kalraiya R, Ingle A and Vaidya M: Implications of cytokeratin $8 / 18$ filament formation in stratified epithelial cells: induction of transformed phenotype. Int $\mathbf{J}$ Cancer 20: 662-668, 2004.

21. Chu YW, Seftor EA, Romer LH and Hendrix MJ: Experimental coexpression of vimentin and keratin intermediate filaments in human melanoma cells augments motility. Am J Pathol 148: 63-69, 1996.

22. Hansson A, Bloor BK, Sarang Z, Haig Y, Morgan PR, Stark HJ, Fusenig NE, Ekstrand J and Grafstrom RC: Analysis of proliferation, apoptosis and keratin expression in cultured normal and immortalized human buccal keratinocytes. Eur J Oral Sci 111: 34-41, 2003.

23. Ranganathan K, Kavitha R, Sawant SS and Vaidya MM: Cytokeratin expression in oral submucous fibrosis - an immunohistochemical study. J Oral Pathol Med 35: 25-32, 2006.

24. Gilbert S, Loranger A, Daigle N and Marceau N: Simple epithelium keratins 8 and 18 provide resistance to Fas-mediated apoptosis. The protection occurs through a receptor-targeting modulation. J Cell Biol 154: 763-773, 2001.

25. Gires O, Mack B, Rauch J and Matthias C: CK8 correlates with malignancy in leukoplakia and carcinomas of the head and neck. Biochem Biophys Res Commun 343: 252-259, 2006.

26. Xu XC, Lee JS, Lippman SM, Ro JY, Hong WK and Lotan R: Increased expression of cytokeratins CK8 and CK19 is associated with head and neck carcinogenesis. Cancer Epidemiol Biomarkers Prev 4: 871-876, 1995.

27. Reibel J: Prognosis of oral pre-malignant lesions: significance of clinical, histopathological and molecular biological characteristics. Crit Rev Oral Biol Med 14: 47-62, 2003.

28. Takeda T, Sugihara K, Hirayama Y, Hirano M, Tanuma JT and Semba I: Immunohistological evaluation of Ki-67, p63, CK 19 and p53 expression in oral epithelial dysplasias. J Oral Pathol Med 35: 369-375, 2006.

29. Su L, Morgan PR and Lane EB: Keratin 14 and 19 expression in normal, dysplastic and malignant oral epithelia. A study using in situ hybridization and immunohistochemistry. J Oral Pathol Med 25: 293-301, 1996.

30. Ogden GR, Lane EB, Hopwood DV and Chisholm DM: Evidence for field change in oral cancer based on cytokeratin expression. Br J Cancer 67: 1324-1330, 1993. 